\title{
Exploring Influencing Factors of Chronic Obstructive Pulmonary Disease Based on Elastic Net and Bayesian Network
}

\section{Dichen Quan}

Shanxi Medical University

Jiahui Ren

Shanxi Medical University

Hao Ren

Shanxi Medical University

\section{Liqin Linghu}

Shanxi Medical University

Xuchun Wang

Shanxi Medical University

Meichen Li

Shanxi Medical University

\section{Yuchao Qiao}

Shanxi Medical University

\section{Zeping Ren}

Shanxi Centre for Disease Control and Prevention

Lixia Qiu ( $\nabla$ qlx_1126@163.com )

Shanxi Medical University

\section{Research Article}

Keywords: Influencing, Chronic Obstructive, Pulmonary Disease, Network

Posted Date: January 3rd, 2022

DOI: https://doi.org/10.21203/rs.3.rs-1163618/v1

License: (c) (i) This work is licensed under a Creative Commons Attribution 4.0 International License.

Read Full License 


\section{Abstract}

This study aimed to construct Bayesian networks(BNs) to analyze the network relationship between those influencing factors and COPD, and to explore their intensity of effect on COPD through network reasoning. Elastic Net and Max-Min Hill-Climbing(MMHC) hybrid algorithm were adopted to screen the variables on the monitoring data of COPD among residents in Shanxi Province, China from 2014 to 2015, and construct BNs respectively. After variables selection by Elastic Net, 10 variables closely related to COPD were selected finally. The BNs constructed by MMHC showed that smoking status, household air pollution, family history, cough, air hunger or dyspnea were directly related to COPD, and Gender was indirectly linked to COPD through smoking status. Moreover, smoking status, household air pollution and family history were the parent nodes of COPD, and cough, air hunger or dyspnea represented the child nodes of COPD. In other words, smoking status, household air pollution and family history were related to the occurrence of COPD, and COPD would make patients' cough, air hunger or dyspnea worse. Generally speaking, BNs could reveal the complex network relationship between COPD and its relevant factors well, making it more convenient to carry out targeted prevention and control of COPD.

\section{Introduction}

Chronic obstructive pulmonary disease (COPD) represents a common disease characterized by persistent respiratory symptoms and airflow limitation. Its clinical manifestations comprise cough, expectoration, chest tightness, air hunger or dyspnea. In severe cases, it would progress into respiratory failure and Corpulmonale, which is a threat to the patients' quality of life. It has emerged as the fifth largest economic burden worldwide ${ }^{1,2}$, especially in developing countries, where COPD owns high morbidity and mortality. One study has shown that about 1 million people die of COPD every year in China, accounting for $30 \%$ of the deaths by COPD globally ${ }^{3}$. Additionally, COPD constitutes the third and fourth leading cause of death in China's rural and urban areas, respectively ${ }^{4}$. Obviously, COPD has become an important public health problem.

To comprehensively analyze its influencing factors and the complex relationship between them, and thus to reduce occurrence is all the more important.

Previously, studies on COPD generally explored the influencing factors of COPD by logistic regression, which required those factors to be inter-independent, and reflected the correlations between factors and COPD based on odds ratio. For example, in 2018, Wang Chen ${ }^{5}$ utilized logistic regression to detect the related risk factors of COPD, suggesting that sex, age, years of smoking, and severe exposure to PM2.5 were significantly associated with COPD. However, in clinical research, correlations often exist in influencing factors of disease. Therefore, it fails to meet the prerequisite of independent variables. In addition, logistic regression is unable to reveal direct or indirect influencing factors of COPD ${ }^{6}$.

Bayesian networks(BNs), proposed by Pearl Judea in 1987, has been widely used ${ }^{7}$. Without strict statistical hypothesis ${ }^{8}, \mathrm{BNs}$ constitute directed acyclic graph (DAG), reflecting potential relationship 
among influencing factors, and conditional probability distribution table (CPT), which demonstrates the correlation intensity among factors ${ }^{9}$. As such, it allows for complex networks between disease and its influencing factors, overcoming the limitation of traditional logistic regression ${ }^{10}$. Moreover, with the information of known nodes, BNs can infer the probability of unknown nodes, flexibly showing the impact of relevant risk factors on $\mathrm{COPD}^{11,12}$.

BNs learning refers to obtaining a complete Bayesian network by existing information. The construction method consists of parameter learning and structure learning ${ }^{13}$. The former one assumes that the network structure is known, then determining the parameters in the network. This article focuses on structure learning, a more commonly-used method, which could be divided into score-based search ${ }^{14}$ and constraint-based algorithm ${ }^{15}$.

The essence of score-based search is to find out the BNs whose score function reaches the maximum. Nonetheless, it's hard ${ }^{16}$ to obtain an optimal network structure under large structure space. The constraint-based algorithm enjoys a high learning efficiency and can obtain global optimal solution, but it also comes with some shortcomings. Firstly, independence of different nodes is sophisticated to judge, and the independence tests between nodes increases exponentially with the increase of nodes. Secondly, the results of high-order conditional independence test are unreliable. Due to their limitations, some scholars have proposed a hybrid algorithm, Max-Min Hill-Climbing(MMHC), a widely used hybrid algorithm, which includes two phases. In the first stage, it builds an undirected framework of BNs to reduce the search space by using constraint-based algorithms. Then the score-based search is used to add, delete and change the direction of edges in the constrained space, to find the network with the highest score ${ }^{16}$. Thus, MMHC hybrid algorithm combines the two algorithms skillfully and overcomes their shortcomings effectively ${ }^{17}$.

Undoubtedly, risk factors for COPD abound, but it's improper to corporate all these factors into BNs. The networks would become extremely complicated and less accurate with more variables. So feature selection is of great necessity. Since Lasso regression does not take into account the correlation between features, it is not suitable for screening variables with multicollinearity. Ridge regression cannot select the model as well, on account of no prediction factor with zero actual coefficients. However, Elastic Net ${ }^{18}$ can $^{2}$ combine the two methods and carry on the feature selection through cross-verification. By adopting Elastic Net, an ideal sparse model can be obtained and the influence of correlation between the observed variables can be compensated.

Hence, we intended to employ Elastic Net to filter influencing factor, which has a strong correlation with COPD, and then used MMHC to build BNs of COPD and its influencing factors, exploring potential relationship between COPD and these factors, so as to provide a theoretical guidance for prevention and reduction of COPD.

\section{Results}


Characteristics of the study population. Among the 2424 initial study participants, 352 subjects with incomplete data were excluded, and 2072 were left for the analysis. Among them, 51.8\% were men and $48.2 \%$ were women. $36.6 \%$ of the participants were aged between 40 and $49,35.0 \%$ were between 50 and $59,23.4 \%$ were between 60 and 69 , and $5.0 \%$ were over 70 (As shown in Table 1). The prevalence of COPD in this study represented $13.4 \%(19.9 \%$ in males and $6.3 \%$ in females). As age increase, the prevalence of COPD increases gradually. As shown in Figure 1, the prevalence of COPD was highest in people older than 70 , reaching $22.3 \%$.

Screening of variables associated with COPD by Elastic Net. 16 risk factors related to COPD were included in the Elastic Net model, and the key parameter values $(\lambda=0.18595, a=0.12)$ for optimizing the model performance were selected by a ten-fold cross-validation. In the end, the coefficients of influencing factors not closely related to COPD would be compressed to 0 and eliminated, and the final 10 variables were obtained, as shown in Table 1. This method was used to determine the factors that had strong correlations with COPD, thus simplifying the structure of later BNs.

Table 1. Selected variables and their regression coefficients

\begin{tabular}{|rrrr|}
\hline Variable & coefficients & Variable & coefficients \\
\hline $\mathrm{x}_{1}$ & 0.24111383 & $\mathrm{x}_{8}$ & 0.07155523 \\
$\mathrm{x}_{2}$ & -0.07398471 & $\mathrm{x}_{10}$ & 0.16883457 \\
$\mathrm{x}_{4}$ & 0.074471365 & $\mathrm{x}_{13}$ & 0.04980561 \\
$\mathrm{x}_{6}$ & 0.22663873 & $\mathrm{x}_{14}$ & 0.22661544 \\
$\mathrm{x}_{7}$ & 0.13507333 & $\mathrm{x}_{15}$ & 0.08082141 \\
\hline
\end{tabular}

Bayesian networks model of COPD. According to the 10 factors selected by Elastic Net in the previous stage, the MMHC algorithm was used to further construct the BNs of COPD and its influencing factors. As shown in Figure 2, a COPD model with 11 nodes and 18 directed edges was constructed. The directed edges represented the dependence of various related factors on COPD. The numbers in the figure represented the prior probability of each node. For example, the prior probability of COPD was 0.134 , that is, $P(C O P D)=0.134$. The results showed that smoking status, household air pollution, family history, cough, air hunger or dyspnea were directly related to COPD. Among them, smoking status, household air pollution, and family history constituted the parent nodes of COPD, that is, they were related to the occurrence of COPD. Cough, air hunger or dyspnea were child nodes of COPD. Namely, COPD was related to the occurrence of Cough, Air hunger or dyspnea.

Reasoning model of COPD. BNs can infer the probability of an unknown node (COPD) based on the state of a known node, and make COPD risk determination possible. If an individual smokes, the 
probability of suffering from COPD is 0.215 , that is, $P(C O P D \mid$ Smoking status $)=0.215$, as shown in Supplementary Fig.S1; if the individual also used wood, animal feces or coal in the past 6 months or more to cook or heat, the probability of suffering from COPD will rise to 0.246 , that is, P(COPD I Smoking status, Household air pollution) $=0.246$, as shown in Supplementary Fig.S2; if the individual has a family history of respiratory disease at the same time, then the possibility of suffering from COPD goes up to 0.280 , that is, $\mathrm{P}(\mathrm{COPD} \mid$ Smoking status, Household air pollution, Family history $)=0.280$, as shown in Supplementary Fig.S3. In the same way, when a person suffers from COPD, the probability of Cough rises from 0.0887 to 0.201 , that is, $P$ (Cough | COPD) $=0.201$, and its probability of Air hunger or dyspnea increases from 0.184 to 0.289 , that is, $P$ (Air hunger or dyspnea | COPD) $=0.289$, as shown in Supplementary Fig.S4.

\section{Discussion}

As the prevalence and morbidity continue to rise, COPD has become an important public health issue. Globally, it is the main cause of disability among elderly population and has become the fifth-largest burden of the global economy ${ }^{2}$. This study showed that the prevalence of COPD in Shanxi Province, China in 2014 was $13.4 \%$, which was comparable to the national COPD prevalence of $13.6 \%$. However, in the past ten years, the prevalence of COPD among residents over 40 in China has increased from $8.2 \%$ in $2002^{19}$ to $13.7 \%$ in $2012^{20}$. This indicated that Shanxi Province should attach importance to the prevention and treatment of COPD.

The BNs constructed by the MMHC algorithm can explore the complex network connections between COPD and its various influencing factors. The results of BNs model showed that smoking status, household air pollution, and family history were directly related to COPD, and gender was indirectly related to COPD through smoking. In addition, the BNs can also describe the relationship between other factors, such as the network relationship between family history, respiratory disease, air hunger or dyspnea, cough, expectoration and other factors, as shown in Figure 2. Logistic regression can't show relationships between variables, because it is a model built on the condition that these factors are interindependent. The conditional probability distribution table of the parent node of COPD was shown in SupplementaryTab.S2. It can be seen that the probability dependence between COPD and the three-parent nodes of smoking status, household air pollution, and family history. If an individual had smoking status, household air pollution, family history at the same time, then he was $28.0 \%$ likely to develop COPD, with $P$ (COPD | smoking status, household air pollution, family history) $=0.280$.

Smoking is currently recognized as the most important risk factor for COPD. The chemicals and fine particles produced during tobacco burning are the main causes of chronic bronchial inflammation and airway obstruction. Su J et a ${ }^{21}$ found smokers were 2.46 times more likely to develop COPD than nonsmokers, after adjusting for other factors. In our study, the smoking rate of residents aged 40 years or older reached $41.4 \%$, in which more than 70 percent of men smoke, reflecting the high prevalence of smoking behavior in the population. From the perspective of COPD prevention and control, tobacco control and non-exposure to tobacco smoke prove one of the most important intervention methods. 
In 2016, $\mathrm{WHO}^{22}$ estimated that about 3.1 billion people in low-and middle-income countries still cooked with contaminated fuel, causing about 4.3 million premature deaths each year, equivalent to $7.7 \%$ of global deaths. It also accounted for one third of all COPD deaths in low - and middle-income countries. It's a common phenomenon for residents to use polluted fuel for cooking or heating. In this survey, household air pollution rate was as high as $69.1 \%$. Long-term exposure to those harmful gases can easily lead to COPD, which is mainly caused by airway reaction. Therefore, residents should change the way of heating and cooking, and the use of clean fuel must be promoted to reduce harmful exposure.

Having a family history of respiratory diseases will increase the incidence of COPD, suggesting that genetic susceptibility is also closely related to COPD. At present, some studies have found that the polymorphisms of a-antitrypsin, matrix metalloprotein, tumor necrosis factor $a$, interleukin and other genes were related to the pathogenesis of COPD, but further research is needed to confirm ${ }^{23-25}$.

In conclusion, Bayesian networks combined with Elastic Net allow for exploration of factors closely related to COPD, and discovery of their complex network relationships with linkage. This approach could provide a new idea for identification of disease-related risk factors, so as to take more targeted measures in disease control and prevention.

\section{Methods}

Study participants. In this study, data were obtained from the COPD monitoring of residents from 2014 to 2015, which was carried out in Shanxi Province, China. After excluding missing data, 2072 valid cases were retained. Based on multi-stage stratified random sampling, the survey was conducted among Chinese residents aged 40 years or older in Taiyuan, Datong, Linfen and Xinzhou of Shanxi Province. The survey included basic information (such as gender, age, cultural level), respiratory symptoms (such as cough, expectoration, air hunger or dyspnea), personal diseases (such as childhood respiratory, hypertension) and risk factors exposure (such as household air pollution, occupational exposure). These factors and their assignments were depicted in SupplementaryTab.S1. This study was approved by the China-japan Friendship Hospital. Informed consent was signed by all study participants or their agents. All experiments and methods were performed in accordance with the relevant guidelines and regulations.

The eligibility criterion for this study was residents of Chinese nationals aged 40 years or older who had lived in the monitoring area for at least 6 months during the 12 months preceding the survey. The exclusion criteria were as follows: (1) residents living in functional areas, such as barracks, military, student dormitories, nursing homes; (2) residents with mental disorders or cognitive disorders, such as dementia, comprehension impairment, deafness; (3) tumor patients found and being treated; (4) residents with high paraplegia; (5) pregnant or lactating women.

Quality control. To ensure the reliability and validity of data, strict measures had been taken in this study. The investigators received standardized professional training before the investigation, and conducted on-site investigation on the survey objects after qualified inspection, and collected relevant 
data through questionnaires. On-site and remote quality control were implemented through synchronous recording. All measuring instruments were calibrated before measurement. All data were entered twice into a database and checked for errors or omissions.

Elastic Net. Regularization is a technique for adding penalties to the objective function. This penalty controls the complexity of the model by reducing the value of the regression coefficient. Elastic Net ${ }^{18}$ is a linear regression model with L1 and L2 norms as regularization matrix. Not only does it retain the characteristic to easily produce feature sparsity like Lasso method, but also inherits the stability of ridge regression. Its algorithm formula is as follows.

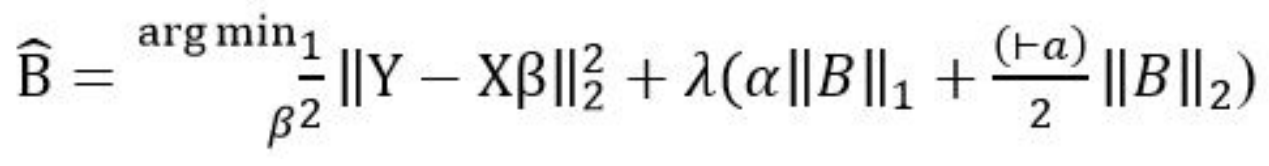

As we can see, $\lambda$ represents the penalty coefficient and $\beta$ is the regression coefficient. For the convex

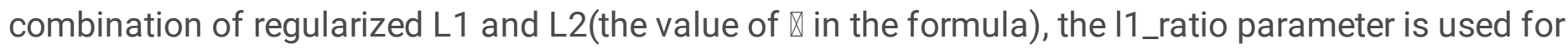
adjustment. The final value of the parameter is selected by ten-fold cross-validation to select the parameter value with the lowest model error.

Bayesian networks. BN is a probability graph model, which can show the probability dependence intensity between factors. It is a directed acyclic graph based on probability theory and graph theory, which consists of nodes representing the variables $U=\left\{x i, \ldots, x_{n}\right\}$ and the directed edges represent the relationship between variables ${ }^{12}$. If the edge from $x_{i}$ to $x_{j}$ exists ${ }^{13}$, then $x_{i}$ is the parent of and $x_{j}$ is the child of $x_{i}$. Each node can quantitatively describe the probability correlation between the node and its parent node through the attached conditional probability distribution table (CPT). In BNs, the formula for calculating the joint probability distribution function of all nodes is as follows.

$$
\begin{gathered}
\mathrm{P}\left(\mathrm{x}_{1}, \mathrm{x}_{2}, \cdots, \mathrm{x}_{\mathrm{n}}\right)=\mathrm{P}\left(\mathrm{x}_{1}\right) \mathrm{P}_{\left(\mathrm{x}_{2} \mid \mathrm{x}_{1}\right)} \cdots \mathrm{P}\left(x_{n \mid x_{1}, x_{2}, \cdots, x_{n-1}}\right) \\
=\prod_{1}^{\mathrm{n}} \mathrm{P}\left(\mathrm{x}_{\mathrm{i}} \mid \pi\left(\mathrm{x}_{\mathrm{i}}\right)\right)
\end{gathered}
$$

$\pi\left(x_{i}\right)$ is the set of parent nodes of $x_{i}, \pi\left(x_{i}\right) \subseteq\left(x_{1}, \ldots, x_{i-1}\right)$. When the value of is known, $\pi\left(x_{i}\right)$ is $x_{i}$ conditionally independent of other variables in $\left(x_{1}, \ldots, x_{i-1}\right)$.

MMHC. MMHC is a widely used Bayesian network hybrid structure learning algorithm, which is mainly divided into two stages. In the first stage, the MMPC algorithm is employed. It can determine the existence of edges without direction, from which the BNs can be constructed. The MMPC algorithm also includes two phases, the first phase starts from the empty set, and then variables input CPC into the empty set successively by using the max-min heuristic function. The first phase doesn't end until all remaining nodes are independent of target node; in the second phase, false positive nodes were deleted 
through the conditional independence test. For a subset of variables $S(S \subseteq C P C)$, if Ind $(X, T \mid S)$ was true, $X$ was deleted from CPC.

In the second stage of the MMHC algorithm, the mountain climbing method is used to locally adjust the current model by adding, deleting and changing the direction of the edges, so as to get several undetermined models, and then calculate the score of each undetermined model to obtain the BNs with the highest score ${ }^{15}$.

Definition. After the bronchodilation test, Participant whose ratio of forced expiratory volume in the first second (FEV1) to forced vital capacity (FVC) was less than 70\%, was determined as COPD patient. The age consisted of four types: $40-49,50-59,60-69, \geq 70$, and cultural level was divided into three levels: junior high school and below, senior high school, college and above. Bodyweight was classified as: underweight $\left(\mathrm{BMI}<18.5 \mathrm{~kg} / \mathrm{m}^{2}\right)$; Normal body weight (BMI 18.5-23.9kg/ $\left.\mathrm{m}^{2}\right)$; Overweight (BMI 24.0$\left.27.9 \mathrm{~kg} / \mathrm{m}^{2}\right)$; Obesity: (BMI $\left.\geq 28.0 \mathrm{~kg} / \mathrm{m}^{2}\right)$. Participants who smoked more than one cigarette a day for the past six months were defined as smokers. Household air pollution referred the use of wood, animal manure or coal for cooking or heating over the past six months or more. Exposure to dust or harmful gases at work (including farm work) was defined as occupational exposure. One or both parents who had suffered from respiratory diseases such as asthma, chronic bronchitis, emphysema, were defined as having a family history of respiratory diseases.

Statistical analysis. Descriptive statistics of influencing factors were performed using IBM SPSS Version 22 (IBM Corp., Armonk, NY, USA). Elastic Net was employed to filter variables in Python 3.7.0. The structure of BNs was constructed by the MMHC in R studio 4.0.5(R Development Core Team), and the maximum likelihood method was used for parameter learning. The drawing of the BNs and reasoning models were realized by Netica (Norsys Software Corp., Vancouver, BC, Canada). Additionally, the maximum likelihood method was used to obtain the values for CPT.

\section{Declarations}

Data availability. The data that support the findings of this study are available from the corresponding author upon reasonable request.

\section{Acknowledgements}

This study was supported by the National Natural Science Foundation of China Project (grant numbers:81973155). The funding body played no role in the design of the study and collection, analysis, and interpretation of data and in writing the manuscript. The authors sincerely thank all teachers in the statistical research office of Shanxi medical university and all interviewers for survey data collection work.

\section{Author Contributions}


D.C.Q., J.H.R. and H.R. analyzed and interpreted the data, and are major contributors in writing the manuscript; L.Q.L.H. were responsible for preprocessing the data and checking the results; Z.P.R. conducted the survey and collected data; X.C.W., M.C.L. and Y.C.Q. were involved in compiling the data and summarizing the results; L.X.Q. gave constructive suggestions for the manuscript. All authors revised the manuscript for important intellectual content and approved the final version.

Competing Interests: The authors declare no competing interests.

\section{References}

1. Martinez, F. J. et al. At the Root: Defining and Halting Progression of Early Chronic Obstructive Pulmonary Disease. 196, 569 (2018).

2. Song, Q., Chen, P. \& Liu, X. M. J. R. R. The role of cigarette smoke-induced pulmonary vascular endothelial cell apoptosis in COPD. 22 (2021).

3. Yin, P. et al. A subnational analysis for mortality and prevalence of chronic obstructive pulmonary disease in China 1990- 2013: Findings from Global Burden of Disease Study (GBD) 2013. 1269-1280 (2016).

4. Zhu, B., Wang, Y., Ming, J., Chen, W. \& Zhang, L. Disease burden of COPD in China: a systematic review. International journal of chronic obstructive pulmonary disease 13, 1353-1364, doi:10.2147/copd.S161555 (2018).

5. Wang, C. et al. Prevalence and risk factors of chronic obstructive pulmonary disease in China (the China Pulmonary Health [CPH] study): a national cross-sectional study.

6. Allison, P. D. J. S. P. Logistic regression using the SAS system: Theory and application. (1999).

7. Koch, D., Eisinger, R. S. \& Gebharter, A. J. J. o. T. B. A causal Bayesian network model of disease progression mechanisms in chronic myeloid leukemia. 433, 94-105 (2017).

8. Ni, W. Q. et al. Serum lipids and associated factors of dyslipidemia in the adult population in Shenzhen. Lipids in health and disease 14, 71, doi:10.1186/s12944-015-0073-7 (2015).

9. Wei, Z. et al. [Using the Tabu-search-algorithm-based Bayesian network to analyze the risk factors of coronary heart diseases]. Zhonghua liu xing bing xue za zhi = Zhonghua liuxingbingxue zazhi 37, 895-899, doi:10.3760/cma.j.issn.0254-6450.2016.06.031 (2016).

10. Kung-Jeng et al. Modeling and predicting the occurrence of brain metastasis from lung cancer by Bayesian network: A case study of Taiwan. (2014).

11. Burnside, E. S., Rubin, D. L. \& Shachter, R. D. Using a Bayesian network to predict the probability and type of breast cancer represented by microcalcifications on mammography. Studies in health technology and informatics 107, 13-17 (2004).

12. Hughes, R. E. Using a Bayesian Network to Predict L5/S1 Spinal Compression Force from Posture, Hand Load, Anthropometry, and Disc Injury Status. Applied bionics and biomechanics 2017, 2014961, doi:10.1155/2017/2014961 (2017). 
13. Kaewprag, P. et al. Predictive models for pressure ulcers from intensive care unit electronic health records using Bayesian networks. 17, 65 (2017).

14. Campos, L. Independency Relationships in Singly Connected Networks. (1994).

15. Heckerman, D., Geiger, D. \& Chickering, D. M. J. M. L. Learning Bayesian Networks: The Combination of Knowledge and Statistical Data. 20, 197-243 (1995).

16. Tsamardinos, I., Brown, L. E. \& Aliferis, C. F. J. M. L. The max-min hill. 65, 31-78 (2006).

17. Haff, I. H., Aas, K., Frigessi, A. \& Lacal, V. Structure learning in Bayesian Networks using regular vines \%J Computational Statistics and Data Analysis. 101 (2016).

18. Zou, H. \& Hastie, T. Addendum: "Regularization and variable selection via the elastic net" [J. R. Stat. Soc. Ser. B Stat. Methodol. 67 (2005), no. 2, 301-320; MR2137327]. journal of the royal statistical society $67,768-768$ (2010).

19. Zhong, N., Chen, W., Yao, W., Chen, P. \& Ran, P. Prevalence of Chronic Obstructive Pulmonary Disease in China: A Large, Population-based Survey. American Journal of Respiratory and Critical Care Medicine 176, 753-760 (2007).

20. Prevalence and risk factors of chronic obstructive pulmonary disease in China (the China Pulmonary Health [CPH] study): a national cross-sectional study. Lancet (2018).

21. Su, J., Ye, Q., Zhang, D., Zhou, J. \& Xu, F. Joint association of cigarette smoking and PM2.5 with COPD among urban and rural adults in regional China. BMC Pulmonary Medicine 21 (2021).

22. Organization, W. H. Burning opportunity: clean household energy for health, sustainable development, and wellbeing of women and children. (2016).

23. Clancy, J. \& Turner, C. Smoking and COPD: the impact of nature-nurture interactions. British Journal of Nursing 22, 820, 822 (2013).

24. Cui, K., Ge, X. Y. \& Ma, H. L. Association of the TNF-a +489 G/A polymorphism with chronic obstructive pulmonary disease risk in Asians: Meta-analysis. Genetics and molecular research: GMR 14, 5210-5220 (2015).

25. Sapey, E., Wood, A. M., Ahmad, A. \& Stockley, R. A. Tumor necrosis factor-\{alpha\} rs361525 polymorphism is associated with increased local production and downstream inflammation in chronic obstructive pulmonary disease. American Journal of Respiratory \& Critical Care Medicine 182, 192-199 (2010).

\section{Figures}




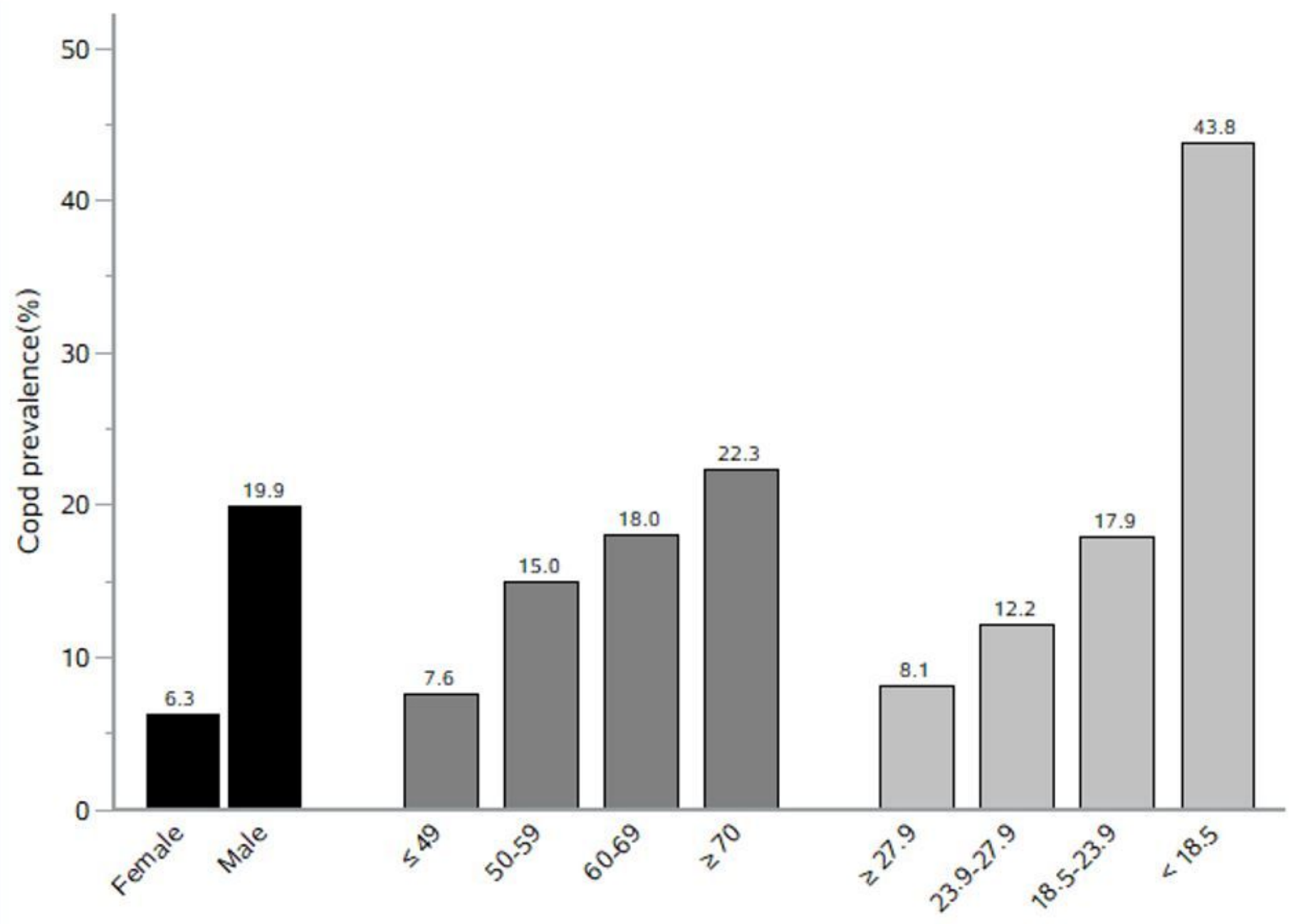

Figure 1

The prevalence of COPD in different gender, age and BMI 


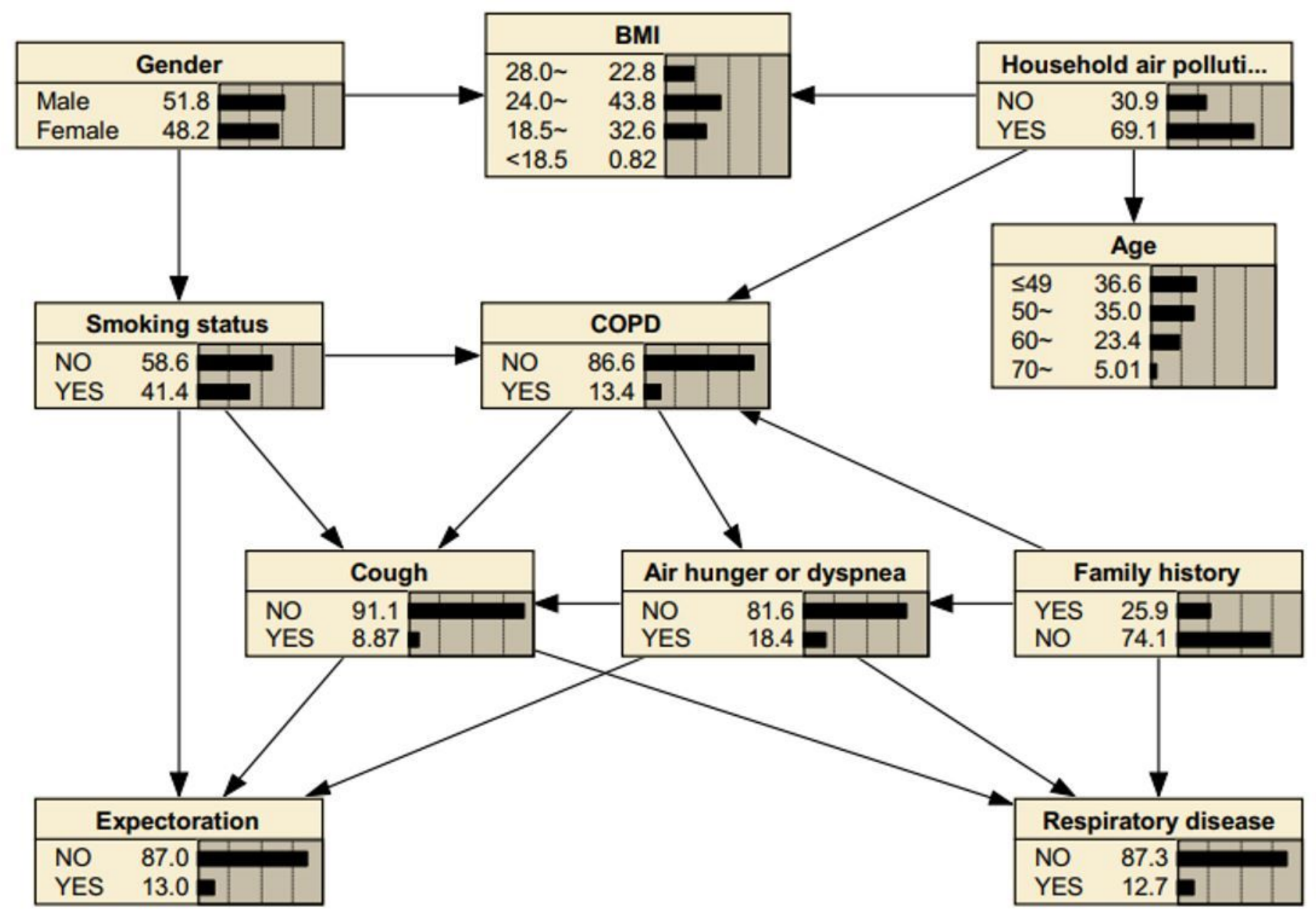

Figure 2

MMHC algorithm to construct COPD Bayesian networks and prior probability

\section{Supplementary Files}

This is a list of supplementary files associated with this preprint. Click to download.

- Supplementarytableandfigure.docx 